\title{
Application of spectroscopic and multispectral imaging technologies on the assessment of ready-to-eat pineapple quality: A performance evaluation study of machine learning models generated from two commercial data analytics tools
}

Evanthia Manthou ${ }^{\mathrm{a}}$, Sergio-Llaneza Lago ${ }^{\mathrm{b}}$, Evaggelos Dagres $^{\mathrm{a}}$, Alexandra Lianou ${ }^{\mathrm{a}}$, Panagiotis Tsakanikas $^{\text {a }}$, Efstathios Panagou ${ }^{\mathrm{a}}$, Maria Anastasiadi $^{\mathrm{b}}$, Fady Mohareb $^{\mathrm{b}}$ \& George -John E. Nychas ${ }^{\mathrm{a}^{*}}$

${ }^{a}$ Laboratory of Microbiology and Biotechnology of Foods, Department of Food Science and Human Nutrition, School of Food \& Nutritional Sciences, Agricultural University of Athens, 11855, Athens, Greece

${ }^{b}$ Bioinformatics Group, Department of Agrifood, School of Water, Energy and Environment Cranfield University, College Road, Cranfield, Bedfordshire, MK43 OAL, UK

*Corresponding author: George-John Nychas; gjn@aua.gr

\begin{abstract}
Recently, rapid, non-invasive analytical methods relying on vibrational spectroscopy and hyper/multispectral imaging, are increasingly gaining popularity in food science. Although such instruments offer a promising alternative to the conventional methods, the analysis of generated data demands complex multidisciplinary approaches based on data analytics tools utilization. Therefore,
\end{abstract}


the objective of this work was to (i) assess the predictive power of different analytical platforms (sensors) coupled with machine learning algorithms in evaluating quality of ready-to-eat (RTE) pineapple (Ananas comosus) and (ii) explore the potentials of The Unscrambler software and the online machine-learning ranking platform, SorfML, in developing the predictive models required by such instruments to assess quality indices. Pineapple samples were stored at $4,8,12^{\circ} \mathrm{C}$ and dynamic temperatures and were subjected to microbiological (total mesophilic microbial populations, TVC) and sensory analysis (colour, odour, texture) with parallel acquisition of spectral data. Fouriertransform infrared, fluorescence (FLUO) and visible sensors, as well as Videometer instrument were used. For TVC, almost all the combinations of sensors and Partial-least squares regression (PLSR) algorithm from both analytics tools reached values of root mean square error of prediction (RMSE) up to $0.63 \log \mathrm{CFU} / \mathrm{g}$, as well as the highest coefficient of determination values $\left(\mathrm{R}^{2}\right)$. Moreover, Linear Support Vector Machine (SVM Linear) combined with each one of the sensors reached similar performance. For odour, FLUO sensor achieved the highest overall performance, when combined with Partial-least squares discriminant analysis (PLSDA) in both platforms with accuracy close to $85 \%$, but also with values of sensitivity and specificity above $85 \%$. The SVM Linear and MSI combination also achieved similar performance. On the other hand, all models developed for colour and texture showed poor prediction performance. Overall, the use of both analytics tools, resulted in similar trends concerning the feasibility of the different analytical platforms and algorithms on quality evaluation of RTE pineapple.

Key words: pineapple, quality, vibrational spectroscopy, multispectral imaging, machine learning, SorfML 


\section{Introduction}

In the context of tremendous technological change, a growing lack of natural resources, and a continuous evolution of consumers' life-styles and consumption habits across the globe, food industry is challenged to provide safe and qualitative food to consumers. To address the need for efficient, safe and environmental respectful production, as well as strict communication and connection with the consumers, several approaches have been developed (Nychas et al., 2016). Among these, analytical methods based on vibrational spectroscopy and hyperspectral / multispectral imaging have gained the attention of scientists, since they could fulfill the needs of food industry as rapid and efficient methods for assessing food quality (Fengou et al., 2019b; Barbin et al., 2015; Papadopoulou et al., 2011; Ammor et al., 2009; Camps \& Christen, 2009), safety (Grewal et al., 2015; Brandily et al., 2011; Davis et al., 2010; Wang et al., 2010) and authentication-adulteration (Huyan et al., 2018; Ropodi et al., 2017; Suhandy et al., 2017; Jacquot et al., 2015; Ropodi et al., 2015; . In contrast with the time-consuming and expensive conventional and molecular-based techniques, the aforementioned approaches constitute a non-destructive and sensible alternative, also suitable for in-, on-, and at-line monitoring (Efenberger-Szmechtyk et al., 2018; Nychas et al., 2016; Kumar et al., 2014). Such tools have been successfully reported in the literature as promising tools for quality and safety assessment of different meat products, such as poultry (Barbin et al., 2015; Grewal et al., 2015), pork and beef (Fengou et al., 2019a; Ropodi et al., 2017; Ropodi et al., 2015; Trinderup et al., 2015; Papadopoulou et al., 2011; Panagou et al., 2014; Ammor et al., 2009; Prieto et al., 2009), as well as fish (Fengou et al., 2019b). Their application on olive oil (Guzmán et al., 2015; de la Mata et al., 2012), cheese (Jacquot et al., 2015; Subramanian et al., 2011), fruits (Liu et al., 2015; Coldea et al., 2013; Unay et al., 2011; Camps \& Christen, 2009; Suhandy et al., 2009) and vegetables (Tsakanikas et al., 2018; Sravan Kumar et al., 2015; Løkke et al., 2013) has also been demonstrated at least at the laboratory scale.

It should be noted that although these instruments/approaches can be considered as efficient, the 
multivariate nature of the sensor output, is rather complex and usually needs processing and/or dimensionality reduction, before the results can be interpreted (Jollife \& Cadima, 2016). Nowadays, in the food sector, a plethora of machine learning approaches has been proposed by different authors in order either to predict or to quantify safety and quality of different foods using fingerprints or other 'omics' data (den Besten et al., 2018; Ropodi et al., 2016). At the same time open sources platforms are contributing in enhancing food safety management system (Tenenhaus-Aziza \& Ellouze, 2015; Nychas et al., 2008).

Indeed, the need of computational tools in the area of food science /microbiology has been recognised, due to their capacity to analyse high volumes of heterogeneous data generated from the innovative technologies (Truong et al., 2019; Granato et al., 2018; Roberts \& Cozzolino, 2016). This trend is clearly followed by the development of various algorithms, among others these include Ordinary Least Squares (OLS), Stepwise Linear modelling (SL), Principal Component Analysis (PCA), Partial Least Squares (PLS), Support Vector Machines (SVM), Random Forests (RF) and kNearest Neighbours $(\mathrm{kNN})$ which can be found either in free or not commercial software with userfriendly and easy-to-use interface. However, choosing the appropriate machine learning approach based on the question that should be addressed, is often challenging and involves a comparative analysis between various algorithms in order to achieve the best possible and realistic performance. This procedure often requires strong statistical and deep interpretation knowledge (Estelles-Lopez et al., 2017).

Even though, the spectroscopic and multispectral imaging techniques have been implemented in a broad range of food products, the application of these technologies to fresh-cut and ready-to-eat (RTE) produces including pineapple (Ananas comosus), is limited, regardless their popularity and market value. Lunadei et al. (2011) evaluated the enzymatic browning of fresh-cut apple slices using multispectral imaging, while a second study of Lunadei et al. (2012) focused on the colour quality of RTE spinach leaves using the same analytical method. Recently, Tsakanikas et al. (2018) studied the 
microbial quality of RTE green salads (rocket and baby spinach) using different non-invasive sensors based on spectroscopy. As far as RTE pineapple is concerned, only Di Egidio et al. (2009) have studied its shelf life using vibrational spectroscopy. Therefore, the aims of this work are (i) to develop mathematics models based on data derived from different analytical instruments to predict the sensory and microbial quality of RTE pineapple, (ii) to compare the models performance and assess the suitability of different algorithms and analytical platforms for monitoring the various features (iii) to explore, the capabilities and the limitations provided by each data analytical tool.

\section{Materials and methods}

\subsection{Samples analyses}

\subsubsection{Sample preparation and storage conditions}

Fresh-cut and RTE pineapple, packed in PVC trays (each containing $220 \mathrm{~g}$ of fruit), was supplied by a local manufacturer in Athens and transported to the laboratory within 24 hours from their production. The pineapples were stored in their original package at three different isothermal temperatures, at $4,8,12^{\circ} \mathrm{C}$ and under dynamic temperature conditions $\left(8\right.$ hours at $4^{\circ} \mathrm{C}, 8$ hours at $8^{\circ} \mathrm{C}$ and 8 hours at $\left.12^{\circ} \mathrm{C}\right)$ in high precision $\left( \pm 0.5^{\circ} \mathrm{C}\right)$ incubators (MIR-153, Sanyo Electric Co., Osaka, Japan). The incubation temperature was recorded at 15-minutes intervals using electronic temperature devices (COX TRACER®, Cox Technologies Inc., Belmont, NC, USA). The sampling was conducted at regular time intervals, depending on the storage temperature, for a maximum period of 10 days. Specifically, the analyses were carried out every 14 and 10 hours ,according to the following sampling time points: $0,14,24,38,48,62,72,86,96,110$ hours, for the first 5 days and every 24 hours until the end of storage. The final time points were 230 hours for $4,8^{\circ} \mathrm{C}$ and the dynamic temperature conditions, while 134 hours for storage at $12^{\circ} \mathrm{C}$.

For each sampling time point, duplicate samples originating from the same temperature conditions but different trays were analyzed. Each sample (tray) was subjected to the following analyses: (i) 
microbiological analysis and pH measurements; (ii) sensory analysis; (iii) Fourier Transform Infrared (FT-IR) spectroscopic measurements; (iv) fluorescence (FLUO) spectroscopic measurements; (v) visible (VIS) spectroscopic measurements and (vi) multispectral image (MSI) acquisition. Different pineapple parts of the same tray were used for the microbiological analysis to prevent any contamination of the samples during the spectroscopic measurements. Four independent storage experiments were finally conducted, using four different batches of pineapple. In the case of the fourth experimental replication and only for FLUO and VIS data, the corresponding spectroscopic measurements were carried out every 24 hours throughout storage. Consequently, the total number of samples for FT-IR and MSI sensors was 424, while for FLUO and VIS was 392.

\subsubsection{Microbiological analysis and pH measurements}

A $25 \mathrm{~g}$ portion of fresh-cut pineapple was aseptically transferred from each tray to a sterile Stomacher bag (Seward Medical, London, UK), diluted with $225 \mathrm{ml}$ of Ringer buffer solution (Lab M Limited, Lanchashire, UK) and homogenized for 60 seconds at $230 \mathrm{rpm}$ in a stomacher device (Lab Blender 400, Seward Medical, London, UK). After the preparation of appropriate serial dilutions with Ringer solution, the total mesophilic microbial populations (TVC) was determined by the spread method on tryptic glycose yeast agar (Plate Count Agar, Biolife, Milan, Italy), after incubation of plates at $25^{\circ} \mathrm{C}$ for 72 hours. The results were expressed as the average ( \pm standard deviation, $n=8) \log$ colony forming units per gram (log CFU/g) of fruit.

The $\mathrm{pH}$ values of fruit samples were measured after the microbiological analysis, using a digital $\mathrm{pH}$ meter (RL150, Russell pH Cork, Ireland) with a glass electrode (Metrohm AG, Herisau, Switzerland). 


\subsubsection{Sensory analysis}

Two staff members evaluated in duplicate the freshness rate of three different sensory features of the samples: odour, colour, and texture. For each sensory parameter, a score was given; 1 for fresh, 2 for intermediate, and 3 for unacceptable. Finally, the samples were classified in two classes: Class 1 for fresh (or acceptable) and Class 2 for non-acceptable pineapple samples. The intermediate samples were also classified in Class 2 to simplify the pipeline process and interpretation, since the samples with score 2 and 3 were commercially unacceptable.

\subsubsection{FT-IR spectroscopy}

In parallel to microbiological analysis, FT-IR spectra were collected using a $\mathrm{ZnSe} 45^{\circ} \mathrm{HATR}$ (Horizontal Attenuated Total Reflectance) crystal (PIKE Technologies, Madison, Wisconsin, United States) on a FT-IR-6200 JASCO spectrometer (Jasco Corp., Tokyo, Japan) equipped with a triglycine-sulphate (TGS) detector and a $\mathrm{Ge} / \mathrm{KBr}$ beamspliter. The samples were cut in small slices of such dimensions in order to cover the crystal and then, they were covered with a piece of aluminum foil. The spectral data were collected over the range of $4000-400 \mathrm{~cm}^{-1}$ at room temperature $\left(22 \pm 2^{\circ} \mathrm{C}\right)$, using the Spectra Manager ${ }^{\mathrm{TM}}$ Code of Federal Regulations (CFR) software version 2 (Jasco Corp.). Reference spectra, called backgrounds, were collected every 4 samples by placing the cleaned blank crystal. For both background and sample readings, 100 scans were accumulated at a nominal resolution of $4 \mathrm{~cm}^{-1}$. The collection time for each sample spectrum was 2 min. At the end of each sampling, the crystal surface was cleaned with detergent, washed with distilled water, dried with lint-free tissue, cleaned with acetone and finally dried with lint-free tissue. The range 1800 and $870 \mathrm{~cm}^{-1}$ was finally used, since this range includes the metabolic activity of spoilage (Di Egidio et al., 2009; Ellis et al., 2002; Al-Jowder et al., 1999). Prior to further analysis, the spectral data were mean-centered and scaled (1/SDEV). Additionally, for TVC prediction, the spectra were subjected to Standard Normal Variate (SNV) pre-processing to provide the most 
important information from FT-IR. On the contrary, no pre-treatments were used for sensory features.

\subsubsection{Multispectral Image analysis}

Multispectral images were captured using the VideometerLab device in 18 different wavelengths ranging from UV (405 nm) to short wave NIR (970 nm) (Carstensen \& Hansen, 2003). The device has been commercialized by Videometer A/S. The spectral radiation is not continuous, but operates at wavelengths $405,435,450,470,505,525,570,590,630,645,660,700,850,870,890,910,940$ and $970 \mathrm{~nm}$. The system is first calibrated radiometrically and geometrically using well-defined standard targets and a light setup is loaded based on the type of the product in each fresh form. The samples of pineapple were placed in a petri dish in a way that covered the entire surface and the dish was placed inside an Ulbricht sphere. The image acquisition and pre-treatment have been described previously in detail (Panagou et al., 2014) and have been implemented using the VideometerLab system software (version2.12.39). For each image, the mean reflectance spectra (along with the standard deviation values) was calculated by averaging the intensity of pixels at each wavelength. Both the mean reflectance values and their standard deviations (in total 36 features) were used for model development, as it is considered that the second ones contain relevant and important information. As it was mentioned before, the spectral data were mean-centered and scaled (1/SDEV), while SNV pre-processing was also performed for odour prediction.

\subsubsection{Visible and fluorescence spectroscopy}

The UV-VIS spectrometer used was the Hamamatsu C12880MA (Hamamatsu Photonics K.K., Shizuoka, Japan). The device has a spectral range from 850 to $340 \mathrm{~nm}$ and spectral resolution of 15 nm. It can be employed either for visible or fluorescence range spectroscopy by switching the mode of spectrometer and changing the settings. Specifically, the scan count was set at 10 and 3, while 
integration time at $250 \mu$ s and $100.000 \mu$ s for acquisition in visible and fluorescence mode, respectively. A UV filter with $400 \mathrm{~nm}$ cutoff wavelength was placed in the front of the spectrometer to obtain spectra only to the visible region. Before the samples measurements, a dark calibration and a reference acquisition are performed. Dark calibration is performed with the light source off placing the spectrometer on a dark surface for both modes. The white reference was performed with the light on using a white material (in our case a folded piece of paper) for visible mode and a non-fluorescent reflective reference standard (a black plastic surface) for fluorescence mode, respectively. The samples were placed in a petri dish covering the entire surface and 10 measurements (absorbance values) were performed in different spots of each sample. The spectral values are expressed as the average of the 10 measurements for each wavelength after a normalization step. For both FLUO and VIS analytical platforms, the range 700-400nm was used for further analysis. The spectra were mean-centered and scaled (1/SDEV). Moreover, in the case of TVC prediction, FLUO spectra were subjected to SNV pre-processing, while VIS spectra to first derivative normalization with a secondorder polynomial and a 9-point window. For texture prediction, FLUO spectra were also subjected to first derivative normalization with a second-order polynomial and a 9-point window.

\subsection{Data analysis}

\subsubsection{The Unscrambler software}

Multivariate data analysis was carried out using the data analytics software, The Unscrambler@ ver. 9.7 (CAMO Software AS, Oslo, Norway). Partial-least squares regression (PLSR) was performed for the correlation between spectral data and microbial counts where, the spectral data were used as independent variables (X) and the TVC as dependent variables (Y). This method is considered suitable for spectroscopic data sets where the dimensionality problem exists (many variables but few samples) and also when the data show strong collinearity and noise (Gromski et al., 2015; Mehmood 
et al., 2012; Wold et al., 2001). For sensory features, since they are categorical variables, Partial-least squares discriminant analysis (PLSDA) was performed (Barker \& Rayens, 2003).

Prior to PLSR/PLSDA, several pre-processing techniques were tested on each data set with the aim to minimize any irrelevant information such as noise, particle size deviations, scattering and drifting effects (Li et al., 2018; Dixit et al., 2017; Suhandy \& Yulia, 2017; Wang et al., 2015). The selected pre-processing procedure was already described in the corresponding section of each analytical method.

The data derived from isothermal storage temperatures were used for the calibration process (training set) and those derived from dynamic temperature conditions for external validation (test set). During the calibration process, leave-one-out cross validation in parallel with Martens uncertainty test was employed in order to eliminate the risk for over-fitting and test the predictive significance of the model, but also to select the significant X -variables (Wold et al., 2001; Westad \& Martens, 2000). The significant independent variables were finally used for the construction of FT-IR and FLUO models for texture assessment.

The prediction performance of the developed PLSR models for each sensor was evaluated based on the following statistical parameters: slope (a), offset (b), correlation coefficient (r), the root mean square error (RMSE), the normalised root mean square error (NRMSE) (Eq.1) and the coefficient of determination $\left(\mathrm{R}^{2}\right.$ ) of the linear regression between the predicted and measured microbiological counts. For PLSDA models, the parameters for performance evaluation were accuracy (Eq. 2), sensitivity (Eq. 3) and specificity (Eq. 4), where positive samples are the fresh or acceptable and negative samples are the non-acceptable samples.

$$
\mathrm{NRMSE}=\frac{\mathrm{RMSE}}{\max (\mathrm{DV})-\min (\mathrm{DV})}
$$

, where DV is the dependent variable (TVC). 


$$
\text { Accuracy }=\frac{\text { samples correctly predicted }}{\text { total number of samples }} \times 100
$$

(Eq. 2)

$$
\text { Sensitivity }=\frac{\text { true positive samples }}{\text { true positive samples }+ \text { false negative samples }} \times 100
$$

$$
\text { Specificity }=\frac{\text { true negative samples }}{\text { true negative samples }+ \text { false positive samples }} \times 100
$$

(Eq. 4)

\subsubsection{SorfML platform}

SorfML is a machine learning classification and regression analysis ranking system (www.SorfML.com). Specifically, it is a free web-platform able to automate the procedure of identifying the best machine learning method for comparing data from several analytical techniques and predict the freshness profiles as well as counts of microorganisms responsible of food spoilage. Using SorfML, users are able to securely upload raw experimental data collected using rapid and/or non-invasive analytical platform (e.g. Multi/Hyper-spectral imaging, Electronic nose, Gas chromatography-Mass spectrometry) in CSV format, and apply various machine learning classification and regression modelling algorithms (e.g. SVM, Neural Network, Random forests) in order to identify the best combination of analytical platform and machine learning algorithm to predict given bacterial species or quality indices. An indicative workflow of the pineapple analysis followed in SorfML is presented in Figure 1.

The algorithms used in this study are k-Nearest Neighbours (kNN) (Silverman \& Jones, 1989), Ranger, a fast version of Random Forest (Wright \& Ziegler, 2017), Linear Support Vector Machine (SVM Linear), Radial Support Vector Machine (SVM Radial) (Boser et al., 1992), PLSR (Wold et al., 2001) and PLSDA (Barker \& Rayens, 2003). In order to generate each model, the dataset was randomly segmented into a training dataset for optimisation, with the $65 \%$ of the total samples, and a 
testing dataset for model validation with the left $35 \%$. The different classes of data were equally represented in training as well as testing data set. The raw data were also centered and scaled, but no other data pre-processing was performed.

The predictive power of the developed models for sensory features was evaluated based on accuracy parameter, but also sensitivity and specificity were provided. For TVC models, RMSE and NRMSE (Eq. 1) parameter for each analytical platform were ranked in heatmaps, while $\mathrm{R}^{2}$ values were also presented. Training performance was assessed using $k$-repeated fold cross-validation with a $\mathrm{k}$ value of 4 and a total number of 10 repetitions to choose the best parameters for each model.

\section{Results}

\subsection{Microbiological spoilage and pH data}

The initial level of TVC (mean \pm standard deviation, $n=8$ ) was $5.09 \pm 0.60 \log$ CFU/g, while the final populations were $7.14 \pm 0.50,7.69 \pm 0.40,7.52 \pm 0.30$ and $7.91 \pm 0.20 \log$ CFU/g during storage at 4 , $8,12^{\circ} \mathrm{C}$ and under dynamic conditions, respectively. The growth was more rapid at the highest temperature.

No significant differences on $\mathrm{pH}$ measurements were found between the different temperatures and during storage. Specifically, the mean (mean \pm standard deviation, $\mathrm{n}=8$ ) initial $\mathrm{pH}$ value was $3.45 \pm 0.05$ and the final values were $3.57 \pm 0.04,3.55 \pm 0.07,3.55 \pm 0.17,3.52 \pm 0.17$ at $4,8,12^{\circ} \mathrm{C}$ and under dynamic conditions, respectively.

\subsection{TVC prediction models}

Concerning TVC, the linear regression between the predicted (estimated) and the measured (observed) TVC values is presented in Table 1 and Figure $\mathbf{2}$ for The Unscrambler, and in Figure 3 for SorfML. The solid lines in Figures $\mathbf{2}$ and $\mathbf{3}$ are the ideal $\mathrm{y}=\mathrm{x}$ lines, while the dashed ones determine the $\pm 1 \log$ unit area. Additionally, SorfML platform provides a ranking of performance 
(with RMSE and NRMSE values) of the various models developed for each analytical platform and machine learning algorithm, which is illustrated in a heatmap plot (Figure 4). In Table 2, the $\mathrm{R}^{2}$ values are also presented for each model developed on SorfML software.

Starting with The Unscrambler, the $\mathrm{a}$ and $\mathrm{b}$ values of the linear regression between the estimated and the observed TVC values have a narrow range from 0.57 to 0.61 and 2.61 to 3.00 , respectively. Additionally, the RMSE and NRMSE values are quite low for all sensors. Actually, the RMSE values are close to $0.5 \log \mathrm{CFU} / \mathrm{g}$. The $\mathrm{R}^{2}$ values are under 0.6 for all the sensors, while the $\mathrm{r}$ value ranges from 0.70 to 0.77 . The FLUO and VIS models exhibit the highest $r$ and $\mathrm{R}^{2}$ values, as well as the lowest RMSE and NRMSE values. The number of components selected for each PLSR model is presented in Table 3 .

In SorfML, RMSE values for all the studied sensors and algorithms were also below 1 log CFU/g. For almost all sensors, PLSR and SVM Linear algorithms exhibit the lowest values of RMSE (and NRMSE), with a range from 0.58 to $0.64 \log \mathrm{CFU} / \mathrm{g}$ and the highest $\mathrm{R}^{2}$ values, with a range from 0.41 to 0.50 . On the other hand, Ranger, $\mathrm{kNN}$ and SVM Radial exhibit the highest RMSE values, above $0.65 \log$ CFU/gr, as well as the lowest $\mathrm{R}^{2}$ values. The regression lines between the estimated and the measured TVC values for non-linear models are not presented in Figure 3. The best model accuracy was achieved through the PLSR model combined with FLUO sensor, showing the lowest RMSE/NRMSE values and the highest $\mathrm{R}^{2}$ values. The tuning parameters selected for each model in SorfML software are presented in Table 4.

\subsection{Sensory prediction models}

Due to the limited data used for sensory analysis, the corresponding results are presented in the Supplementary section. Table $\mathbf{S 1}$ presents the number of acceptable and non-acceptable (in terms of freshness) samples for the three sensory features. It should be noted that for colour and texture, the number of fresh samples are four and three times higher than that of non-acceptable, while for odour, 
the samples are more equally distributed to both classes. The prediction performance of the models developed for each one of the tested analytical platforms are summarized in Table S2 for The Unscrambler. The accuracy ranking of models generated for sensory features on SorfML software is illustrated in Figures S1A-C, while Table S3 provides also the corresponding values of sensitivity and specificity.

For The Unscrambler, none of the models reaches $90 \%$ of accuracy. The models for texture prediction have the lowest accuracy values, while for colour and odour, exceed $80 \%$ for almost all the sensors. However, in the case of colour, the satisfactory accuracy values of models are not followed by similar sensitivity and specificity values. As far as the odour prediction is concerned, , FLUO model is the only model, which has the highest values of both sensitivity (88.46\%) and specificity (85.90\%), and also the highest value of accuracy (86.54\%) among the analytical platforms.

In SorfML, the accuracy percentage of all the models generated for all of the three features, do not exceed $89.23 \%$. As far as the colour and texture is concerned, the models with accuracy values above $80 \%$, show also high sensitivity and low specificity. For odour, FLUO combined with PLSDA, SVM Radial and SVM Linear, but also MSI combined with SVM Linear, show the highest accuracy values. The combinations with the best accuracy, as well as, sensitivity and specificity values are FLUO and PLSDA, together with MSI and SVM Linear.

\section{Discussion}

The introduction of spectroscopic and optical sensing (computer vision) methods in food science and their growing application in a wide range of food products is becoming a clear trend during the last decade. Although these analytical techniques offer rapid and in many cases efficient answers in a non-destructive way, the manipulation of the data and the interpretation of results are still a great challenge (Zhou et al., 2019). 
Data analysis tools is a set of technology, that enable users to analyze and visualize data in order to identify trends and correlations with the goal of supporting decision making. Therefore, data analysis software is considered to be a central requirement for any sector / business (Vassakis et al., 2018). Although a large number of tools are available nowadays, only a limited number of these have proven to be useful for food science. The two applied statistical software, The Unscrambler and SorfML, provide a user-friendly and easy-to-use interface for analyzing large experimental datasets. These automated platforms also provide the opportunity for scientists, let alone food microbiologists with limited statistical and mathematical knowledge, to perform the challenging task of data mining and predictive modelling. However, the users are responsible for learning the advantages and the limitations of each platform and also realize that conflicting outputs are even possible (Nunes et al., 2015).

The Unscrambler software provides the option of limited in number and only linear algorithms, namely PLSR and PLSDA. On the other hand, SorfML platform offers the wide option of choosing linear and non-linear algorithms often used in data analysis. The approach of using different algorithms for the same data, allows comparing the performance of each created model and evaluate their suitability for different scenarios (Estelles-Lopez et al., 2017). Apart from algorithms, the big differences between the two data analytics tools lie on the segmentation of data in training and prediction set, the pre-processing of data and the cross-validation method.

Starting with data segmentation on The Unscrambler, the testing of the models was performed on samples stored in dynamic temperature conditions, since these data include the information from all temperatures and are also considered as a simulation of real life in the food supply chain (Tsakanikas et al., 2018). On the other hand, in SorfML, the data were randomly segmented ensuring that all temperature and storage time groups were equally represented in both data sets and finally provided a less biased selection of sample. Furthemore, using The Unscrambler, various pre-processing methods were tested and those with the best results were finally applied. The purpose was to remove 
the irrelevant information from data and facilitate their interpretation. However, in SorfML, the data were subjected to the minimum treatment, with the aim not to lose important information. As far as the cross validation was concerned, leave-one-out (or full-cross) was used in the The Unscrambler, while k-fold cross-validation used in SorfML. Full-cross validation is a common method, where all samples are used in an exhaustive way providing repeatability of the results, but also may lead to over-optimistic results. On the other hand, a k-fold partitioning could result in folds where samples are not represented in an equal manner (Ropodi et al., 2016).

Besides all these different approaches, the summarized results indicate similar trends about sensors and algorithms ability to assess the quality of RTE pineapple. Specifically, for TVC assessment, all the sensors combined with PLSR algorithm show satisfactory performance in both The Unscrambler and SorfML. In The Unscrambler, the best models with slight differences compared to the others, are that based on FLUO and VIS data. In SorfML, FLUO and PLSR combination also exhibits the best performance with slight differences compared to the other PLSR models.. Apart from PLSR algorithm, it is indicative that SVM Linear combined with every sensor is also appropriate for TVC prediction. Contrarily, non-linear algorithms, tested in SorfML, do not manage to predict the spoilage of RTE pineapple.

An important observation on these results is that the $\mathrm{R}^{2}$ values are quite low, even for the best model performances. It could be argued that according to these low $\mathrm{R}^{2}$ values the prediction performance is poor. However, despite the widely held belief for the usefulness of coefficient of determination, there is no guarantee that a high value of this parameter is indicative of 'goodness of fit'. The value of $\mathrm{R}^{2}$ strongly depends on the width of the prediction interval and the variability present in the data (Granato et al., 2014). In food microbiology, 0.5 log deviations are common even within the same laboratory but also, RMSE values under $1 \log \mathrm{CFU} / \mathrm{g}$, is a rather acceptable result for food microbiology applications. Moreover, the variability inside the experimental replications (batches), as well as between the two biological replicates (duplicate samples) of the same experimental 
replication is too high. The latter issue is very common in plant origin products due to the strong impact of various factors such as, cultivar, geographical region, and agricultural practices. For sensory features, the results were presented in order to reveal a potential trend which would be helpful for further investigation. The results derived from the two platforms show similar conclusions. Regarding the low number of non-acceptable over the fresh samples for colour and texture, the models generated by both software are biased over the fresh samples. Consequently, the presence of a data set with a more balanced proportion of the two classes, could possibly result in better model performances and safer conclusions for colour and texture assessment. For odour, FLUO sensor and PLSDA algorithm emerged as one of the most appropriate combinations for both tools. Additionally, SorfML software indicates that the combination of MSI and SVM Linear may be also appropriate for odour assessment in pineapple.

To conclude, the implementation of different data analytics tools requires wide knowledge of their range of applications and limitations and the results should always be evaluated critically. In this study, the results indicate that both The Unscrambler and SorfML revealed similar trends for the various analytical platforms. Specifically, the assessment of pineapple spoilage could be potentially achieved by the various types of vibrational spectroscopy (FTIR, FLUO and VIS) as well as, multispectral imaging. As far as the sensory features are concerned, the odour, could be possibly assessed by FLUO spectroscopy, conducting a more complete and representative analysis. . It is also indicative that the possibility of testing various algorithms may lead to new options and more reliable results. However, further research including feature selection analysis and data fusion strategies may be crucial in developing even more robust and accurate models (Tsakanikas et al., 2018).

\section{Acknowledgments}

This work has been supported by the project "PhasmaFOOD”, funded from the European Union's Horizon 2020 research and innovation programme under grant agreement No 732541. 


\section{References}

Al-Jowder, O., Defernez, M., Kemsley, E.K., Wilson, R.H., 1999. Mid-infrared spectroscopy and chemometrics for the authentication of meat products. J. Agric. Food Chem. 47, 3210-3218. https://doi.org/10.1021/jf981196d.

Ammor, M.S., Argyri, A., Nychas, G.J.E., 2009. Rapid monitoring of the spoilage of minced beef stored under conventionally and active packaging conditions using Fourier transform infrared spectroscopy in tandem with chemometrics. Meat Sci. 81, 507-514. https://doi.org/10.1016/j.meatsci.2008.10.015.

Barbin, D.F., Kaminishikawahara, C.M., Soares, A.L., Mizubuti, I.Y., Grespan, M., Shimokomaki, M., Hirooka, E.Y., 2015. Prediction of chicken quality attributes by near infrared spectroscopy. Food Chem. 168, 554-560. https://doi.org/10.1016/j.foodchem.2014.07.101

Barker, M., Rayens, W., 2003. Partial least squares for discrimination. J. Chemom. 17, 166-173. https://doi.org/10.1002/cem.785

Boser, B.E., Guyon, I.M., Vapnik, V.N., 1992. Training algorithm for optimal margin classifiers. Proc. Fifth Annu. ACM Work. Comput. Learn. Theory 144-152. https://doi.org/10.1145/130385.130401

Brandily, M.L., Monbet, V., Bureau, B., Boussard-Plédel, C., Loréal, O., Adam, J.L., Sire, O., 2011. Identification of foodborne pathogens within food matrices by IR spectroscopy. Sensors Actuators, B Chem. 160, 202-206. https://doi.org/10.1016/j.snb.2011.07.034

Camps, C., Christen, D., 2009. Non-destructive assessment of apricot fruit quality by portable visible-near infrared spectroscopy. LWT - Food Sci. Technol. 42, 1125-1131. https://doi.org/10.1016/j.lwt.2009.01.015

Carstensen, J.M., Hansen, J.F., 2003. An apparatus and a method of recording an image of an object. Patent family EP1051660.

Coldea, T.E., Socaciu, C., Fetea, F., Ranga, F., Pop, R.M., Florea, M., 2013. Rapid quantitative 
analysis of ethanol and prediction of methanol content in traditional fruit brandies from romania, using FTIR spectroscopy and chemometrics. Not. Bot. Horti Agrobot. Cluj-Napoca 41, 143-149. https://doi.org/10.15835/nbha4119000

Davis, R., Irudayaraj, J., Reuhs, B.L., Mauer, L.J., 2010. Detection of E. coli O157:H7 from ground beef using fourier transform infrared (FT-IR) spectroscopy and chemometrics. J. Food Sci. 75, 340-346. https://doi.org/10.1111/j.1750-3841.2010.01686.x

de la Mata, P., Dominguez-Vidal, A., Bosque-Sendra, J.M., Ruiz-Medina, A., Cuadros-Rodríguez, L., Ayora-Cañada, M.J., 2012. Olive oil assessment in edible oil blends by means of ATR-FTIR and chemometrics. Food Control 23, 449-455. https://doi.org/10.1016/j.foodcont.2011.08.013 den Besten, H.M.W., Amézquita, A., Bover-Cid, S., Dagnas, S., Ellouze, M., Guillou, S., Nychas, G., O’Mahony, C., Pérez-Rodriguez, F., Membré, J.M., 2018. Next generation of microbiological risk assessment: Potential of omics data for exposure assessment. Int. J. Food Microbiol. 287, 18-27. https://doi.org/10.1016/j.ijfoodmicro.2017.10.006

Di Egidio, V., Sinelli, N., Limbo, S., Torri, L., Franzetti, L., Casiraghi, E., 2009. Evaluation of shelflife of fresh-cut pineapple using FT-NIR and FT-IR spectroscopy. Postharvest Biol. Technol. 54, 87-92. https://doi.org/10.1016/j.postharvbio.2009.06.006

Dixit, Y., Casado-Gavalda, M.P., Cama-Moncunill, R., Cama-Moncunill, X., Markiewicz-Keszycka, M., Cullen, P.J., Sullivan, C., 2017. Developments and Challenges in Online NIR Spectroscopy for Meat Processing. Compr. Rev. Food Sci. Food Saf. 16, 1172-1187. https://doi.org/10.1111/1541-4337.12295

Efenberger-Szmechtyk, M., Nowak, A., Kregiel, D., 2018. Implementation of chemometrics in quality evaluation of food and beverages. Crit. Rev. Food Sci. Nutr. 58, 1747-1766. https://doi.org/10.1080/10408398.2016.1276883

Ellis, D.I., Broadhurst, D., Kell, D.B., Jem, J., Goodacre, R., Rowland, J.J., 2002. Rapid and Quantitative Detection of the Microbial Spoilage of Meat by Fourier Transform Infrared 
Spectroscopy and Machine Learning. Appl. Environ. Microbiol. 68, 2822-2828. https://doi.org/10.1128/AEM.68.6.2822

Estelles-Lopez, L., Ropodi, A., Pavlidis, D., Fotopoulou, J., Gkousari, C., Peyrodie, A., Panagou, E., Nychas, G.J., Mohareb, F., 2017. An automated ranking platform for machine learning regression models for meat spoilage prediction using multi-spectral imaging and metabolic profiling. Food Res. Int. 99, 206-215. https://doi.org/10.1016/j.foodres.2017.05.013

Fengou, L.-C., Spyrelli, E., Lianou, A., Tsakanikas, P., Panagou, E.Z., Nychas, G.-J.E., 2019a. Estimation of Minced Pork Microbiological Spoilage through Fourier Transform Infrared and Visible Spectroscopy and Multispectral Vision Technology. Foods 8, 238.

https://doi.org/10.3390/foods8070238

Fengou, L.C., Lianou, A., Tsakanikas, P., Gkana, E.N., Panagou, E.Z., Nychas, G.J.E., 2019b. Evaluation of Fourier transform infrared spectroscopy and multispectral imaging as means of estimating the microbiological spoilage of farmed sea bream. Food Microbiol. 79, 27-34. https://doi.org/10.1016/j.fm.2018.10.020

Granato, D., de Araújo Calado, V.Ô.M., Jarvis, B., 2014. Observations on the use of statistical methods in Food Science and Technology. Food Res. Int. 55, 137-149. https://doi.org/10.1016/j.foodres.2013.10.024

Granato, D., Putnik, P., Kovačević, D.B., Santos, J.S., Calado, V., Rocha, R.S., Cruz, A.G. Da, Jarvis, B., Rodionova, O.Y., Pomerantsev, A., 2018. Trends in Chemometrics: Food Authentication, Microbiology, and Effects of Processing. Compr. Rev. Food Sci. Food Saf. 17, 663-677. https://doi.org/10.1111/1541-4337.12341

Grewal, M.K., Jaiswal, P., Jha, S.N., 2015. Detection of poultry meat specific bacteria using FTIR spectroscopy and chemometrics. J. Food Sci. Technol. 52, 3859-3869. https://doi.org/10.1007/s13197-014-1457-9

Gromski, P.S., Muhamadali, H., Ellis, D.I., Xu, Y., Correa, E., Turner, M.L., Goodacre, R., 2015. A 
tutorial review: Metabolomics and partial least squares-discriminant analysis - a marriage of convenience or a shotgun wedding. Anal. Chim. Acta 879, 10-23.

$\underline{\text { https://doi.org/10.1016/j.aca.2015.02.012 }}$

Guzmán, E., Baeten, V., Pierna, J.A.F., García-Mesa, J.A., 2015. Determination of the olive maturity index of intact fruits using image analysis. J. Food Sci. Technol. 52, 1462-1470. https://doi.org/10.1007/s13197-013-1123-7

Huyan, Z., Ding, S., Liu, X., Yu, X., 2018. Authentication and adulteration detection of peanut oils of three flavor types using synchronous fluorescence spectroscopy. Anal. Methods 10, 32073214. https://doi.org/10.1039/c8ay00837j

Jacquot, S., Karoui, R., Abbas, K., Lebecque, A., Bord, C., Aït-Kaddour, A., 2015. Potential of Multispectral Imager to Characterize Anisotropic French PDO Cheeses: A Feasibility Study. Int. J. Food Prop. 18, 213-230. https://doi.org/10.1080/10942912.2013.828746

Jollife, I.T., Cadima, J., 2016. Principal component analysis: A review and recent developments. Philos. Trans. R. Soc. A Math. Phys. Eng. Sci. 374. https://doi.org/10.1098/rsta.2015.0202

Kumar, N., Bansal, A., Sarma, G.S., Rawal, R.K., 2014. Chemometrics tools used in analytical chemistry: An overview. Talanta 123, 186-199. https://doi.org/10.1016/j.talanta.2014.02.003

Li, B., Lecourt, J., Bishop, G., 2018. Advances in non-destructive early assessment of fruit ripeness towards defining optimal time of harvest and yield prediction —a review. Plants 7, 1-20. $\underline{\text { https://doi.org/10.3390/plants7010003 }}$

Liu, C., Liu, W., Chen, W., Yang, J., Zheng, L., 2015. Feasibility in multispectral imaging for predicting the content of bioactive compounds in intact tomato fruit. Food Chem. 173, 482-488. https://doi.org/10.1016/j.foodchem.2014.10.052

Løkke, M.M., Seefeldt, H.F., Skov, T., Edelenbos, M., 2013. Color and textural quality of packaged wild rocket measured by multispectral imaging. Postharvest Biol. Technol. 75, 86-95. https://doi.org/10.1016/j.postharvbio.2012.06.018 
Lunadei, L., Diezma, B., Lleó, L., Ruiz-Garcia, L., Cantalapiedra, S., Ruiz-Altisent, M., 2012.

Monitoring of fresh-cut spinach leaves through a multispectral vision system. Postharvest Biol. Technol. 63, 74-84. https://doi.org/10.1016/j.postharvbio.2011.08.004

Lunadei, L., Galleguillos, P., Diezma, B., Lleó, L., Ruiz-Garcia, L., 2011. A multispectral vision system to evaluate enzymatic browning in fresh-cut apple slices. Postharvest Biol. Technol. 60, 225-234.https://doi.org/10.1016/j.postharvbio.2011.02.001

Mehmood, T., Liland, K.H., Snipen, L., Sæbø, S., 2012. A review of variable selection methods in Partial Least Squares Regression. Chemom. Intell. Lab. Syst. 118, 62-69.

https://doi.org/10.1016/j.chemolab.2012.07.010

Nunes, C.A., Alvarenga, V.O., de Souza Sant'Ana, A., Santos, J.S., Granato, D., 2015. The use of statistical software in food science and technology: Advantages, limitations and misuses. Food Res. Int. 75, 270-280. https://doi.org/10.1016/j.foodres.2015.06.011

Nychas, G.J.E., Panagou, E.Z., Mohareb, F., 2016. Novel approaches for food safety management and communication. Curr. Opin. Food Sci. 12, 13-20.

https://doi.org/10.1016/j.cofs.2016.06.005

Nychas, G.J.E., Skandamis, P.N., Tassou, C.C., Koutsoumanis, K.P., 2008. Meat spoilage during distribution. Meat Sci. 78, 77-89. https://doi.org/10.1016/j.meatsci.2007.06.020

Panagou, E.Z., Papadopoulou, O., Carstensen, J.M., Nychas, G.J.E., 2014. Potential of multispectral imaging technology for rapid and non-destructive determination of the microbiological quality of beef filets during aerobic storage. Int. J. Food Microbiol. 174, 1-11.

https://doi.org/10.1016/j.ijfoodmicro.2013.12.026

Papadopoulou, O., Panagou, E.Z., Tassou, C.C., Nychas, G.J.E., 2011. Contribution of Fourier transform infrared (FTIR) spectroscopy data on the quantitative determination of minced pork meat spoilage. Food Res. Int. 44, 3264-3271. https://doi.org/10.1016/j.foodres.2011.09.012 Prieto, N., Ross, D.W., Navajas, E.A., Nute, G.R., Richardson, R.I., Hyslop, J.J., Simm, G., Roehe, 
R., 2009. On-line application of visible and near infrared reflectance spectroscopy to predict chemical-physical and sensory characteristics of beef quality. Meat Sci. 83, 96-103. https://doi.org/10.1016/j.meatsci.2009.04.005

Roberts, J.J., Cozzolino, D., 2016. An Overview on the Application of Chemometrics in Food Science and Technology_An Approach to Quantitative Data Analysis. Food Anal. Methods 9, 3258-3267. https://doi.org/10.1007/s12161-016-0574-7

Ropodi, A.I., Panagou, E.Z., Nychas, G.J.E., 2017. Multispectral imaging (MSI): A promising method for the detection of minced beef adulteration with horsemeat. Food Control 73, 57-63. https://doi.org/10.1016/j.foodcont.2016.05.048

Ropodi, A.I., Panagou, E.Z., Nychas, G.J.E., 2016. Data mining derived from food analyses using non-invasive/non-destructive analytical techniques; determination of food authenticity, quality \& safety in tandem with computer science disciplines. Trends Food Sci. Technol. 50, 11-25. https://doi.org/10.1016/j.tifs.2016.01.011

Ropodi, A.I., Pavlidis, D.E., Mohareb, F., Panagou, E.Z., Nychas, G.J.E., 2015. Multispectral image analysis approach to detect adulteration of beef and pork in raw meats. Food Res. Int. 67, 12 18. https://doi.org/10.1016/j.foodres.2014.10.032

Silverman, B.W., Jones, M.C., 1989. E. fix and jl hodges (1951): An important contribution to nonparametric discriminant analysis and density estimation: Commentary on fix and hodges (1951). Int. Stat. Rev. 57, 233-247.

Sravan Kumar, S., Manoj, P., Giridhar, P., 2015. Fourier transform infrared spectroscopy (FTIR) analysis, chlorophyll content and antioxidant properties of native and defatted foliage of green leafy vegetables. J. Food Sci. Technol. 52, 8131-8139. https://doi.org/10.1007/s13197-015$\underline{1959-0}$

Subramanian, A., Alvarez, V.B., Harper, W.J., Rodriguez-Saona, L.E., 2011. Monitoring amino acids, organic acids, and ripening changes in Cheddar cheese using Fourier-transform infrared 
spectroscopy. Int. Dairy J. 21, 434-440. https://doi.org/10.1016/j.idairyj.2010.12.012

Suhandy, D., 2009. Nondestructive measurement of soluble solids content in pineapple fruit using short wavelength near infrared (SW-NIR) spectroscopy. Int. J. Appl. Eng. Res. 4, 107-114.

Suhandy, D., Yulia, M., 2017. The Use of Partial Least Square Regression and Spectral Data in UVVisible Region for Quantification of Adulteration in Indonesian Palm Civet Coffee. Int. J. Food Sci. 2017. https://doi.org/10.1155/2017/6274178

Tenenhaus-Aziza, F., Ellouze, M., 2015. Software for predictive microbiology and risk assessment: A description and comparison of tools presented at the ICPMF8 Software Fair. Food Microbiol. 45, 290-299. https://doi.org/10.1016/j.fm.2014.06.026

Trinderup, C.H., Dahl, A., Jensen, K., Carstensen, J.M., Conradsen, K., 2015. Comparison of a multispectral vision system and a colorimeter for the assessment of meat color. Meat Sci. 102, 1-7. https://doi.org/10.1016/j.meatsci.2014.11.012

Truong, V.K., Dupont, M., Elbourne, A., Gangadoo, S., Pathirannahalage, P.R., Cheeseman, S., Chapman, J., Cozzolino, D., 2019. From academia to reality check: A theoretical framework on the use of chemometric in food sciences. Foods 8. https://doi.org/10.3390/foods8050164

Tsakanikas, P., Fengou, L.C., Manthou, E., Lianou, A., Panagou, E.Z., Nychas, G.J.E., 2018. A unified spectra analysis workflow for the assessment of microbial contamination of ready-to-eat green salads: Comparative study and application of non-invasive sensors. Comput. Electron. Agric. 155, 212-219. https://doi.org/10.1016/j.compag.2018.10.025

Unay, D., Gosselin, B., Kleynen, O., Leemans, V., Destain, M.F., Debeir, O., 2011. Automatic grading of Bi-colored apples by multispectral machine vision. Comput. Electron. Agric. 75, 204-212. https://doi.org/10.1016/j.compag.2010.11.006

Vassakis, K., Petrakis, E., Kopanakis, I., 2018. Big Data Analytics: Applications, Prospects and Challenges Mobile In: Skourletopoulos, G., Mastorakis, G., Mavromoustakis, C., Dobre, C., Pallis, E. (Eds.), Mobile Big Data, A Roadmap from Models to Technologies 10, Springer 
International Publishing. https://doi.org/10.1007/978-3-319-67925-9

Wang, H., Peng, J., Xie, C., Bao, Y., He, Y., 2015. Fruit quality evaluation using spectroscopy technology: A review. Sensors (Switzerland) 15, 11889-11927.

https://doi.org/10.3390/s150511889

Wang, J., Kim, K.H., Kim, S., Kim, Y.S., Li, Q.X., Jun, S., 2010. Simple quantitative analysis of Escherichia coli K-12 internalized in baby spinach using Fourier Transform Infrared spectroscopy. Int. J. Food Microbiol. 144, 147-151. https://doi.org/10.1016/j.ijfoodmicro.2010.09.013

Westad, F., Martens, H., 2000. Variable selection in near infrared spectroscopy based on significance testing in partial least squares regression. J. Near Infrared Spectrosc. 8, 117-124. https://doi.org/10.1255/jnirs.271

Wold, S., Sjöström, M., Eriksson, L., 2001. PLS-regression: A basic tool of chemometrics. Chemom. Intell. Lab. Syst. 58, 109-130. https://doi.org/10.1016/S0169-7439(01)00155-1

Wright, M.N., Ziegler, A., 2017. Ranger: A fast implementation of random forests for high dimensional data in C++ and R. J. Stat. Softw. 77, 1-17. https://doi.org/10.18637/jss.v077.i01

Zhou, L., Zhang, C., Liu, F., Qiu, Z., He, Y., 2019. Application of Deep Learning in Food: A Review. Compr. Rev. Food Sci. Food Saf. 18, 1793-1811. https://doi.org/10.1111/1541-4337.12492

Zhu, X., Li, S., Shan, Y., Zhang, Z., Li, G., Su, D., Liu, F., 2010. Detection of adulterants such as sweeteners materials in honey using near-infrared spectroscopy and chemometrics. J. Food Eng. 101, 92-97. https://doi.org/10.1016/j.jfoodeng.2010.06.014 


\begin{tabular}{lcccccc}
\hline Sensor & $a$ & $b$ & $r$ & RMSE & NRMSE & $\mathrm{R}^{2}$ \\
\hline FT-IR & 0.61 & 2.61 & 0.70 & 0.59 & 13.43 & 0.43 \\
MSI & 0.61 & 2.78 & 0.74 & 0.53 & 12.06 & 0.54 \\
FLUO & 0.59 & 2.96 & 0.77 & 0.51 & 11.61 & 0.58 \\
VIS & 0.57 & 3.00 & 0.77 & 0.51 & 11.61 & 0.58 \\
\hline
\end{tabular}

Table 1. Performance metrics of the PLSR models, based on the different analytical platforms (sensors), for the TVC prediction of RTE pineapple on The Unscrambler software. (a: slope, b: offset, r: correlation coefficient, RMSE: root mean square error (log CFU/g), NRMSE: normalised root mean square error $(\%), \mathrm{R}^{2}$ : coefficient of determination) 


\begin{tabular}{|c|c|c|}
\hline Sensor & Algorithm & $\mathrm{R}^{2}$ \\
\hline \multirow{5}{*}{ FT-IR } & Knn & 0.07 \\
\hline & Ranger & 0.16 \\
\hline & SVM Linear & 0.42 \\
\hline & SVM Radial & 0.12 \\
\hline & PLSR & 0.42 \\
\hline \multirow{5}{*}{ MSI } & Knn & 0.09 \\
\hline & Ranger & 0.13 \\
\hline & SVM Linear & 0.44 \\
\hline & SVM Radial & 0.12 \\
\hline & PLSR & 0.41 \\
\hline \multirow{5}{*}{ FLUO } & Knn & 0.22 \\
\hline & Ranger & 0.19 \\
\hline & SVM Linear & 0.46 \\
\hline & SVM Radial & 0.39 \\
\hline & PLSR & 0.5 \\
\hline \multirow{5}{*}{ VIS } & Knn & 0.08 \\
\hline & Ranger & 0.21 \\
\hline & SVM Linear & 0.48 \\
\hline & SVM Radial & 0.26 \\
\hline & PLSR & 0.27 \\
\hline
\end{tabular}

Table 2. The coefficient of determination $\left(\mathrm{R}^{2}\right)$ of the models derived from the various combinations of the different analytical platforms (sensors) and algorithms, for the TVC prediction of RTE pineapple on SorfML software. 


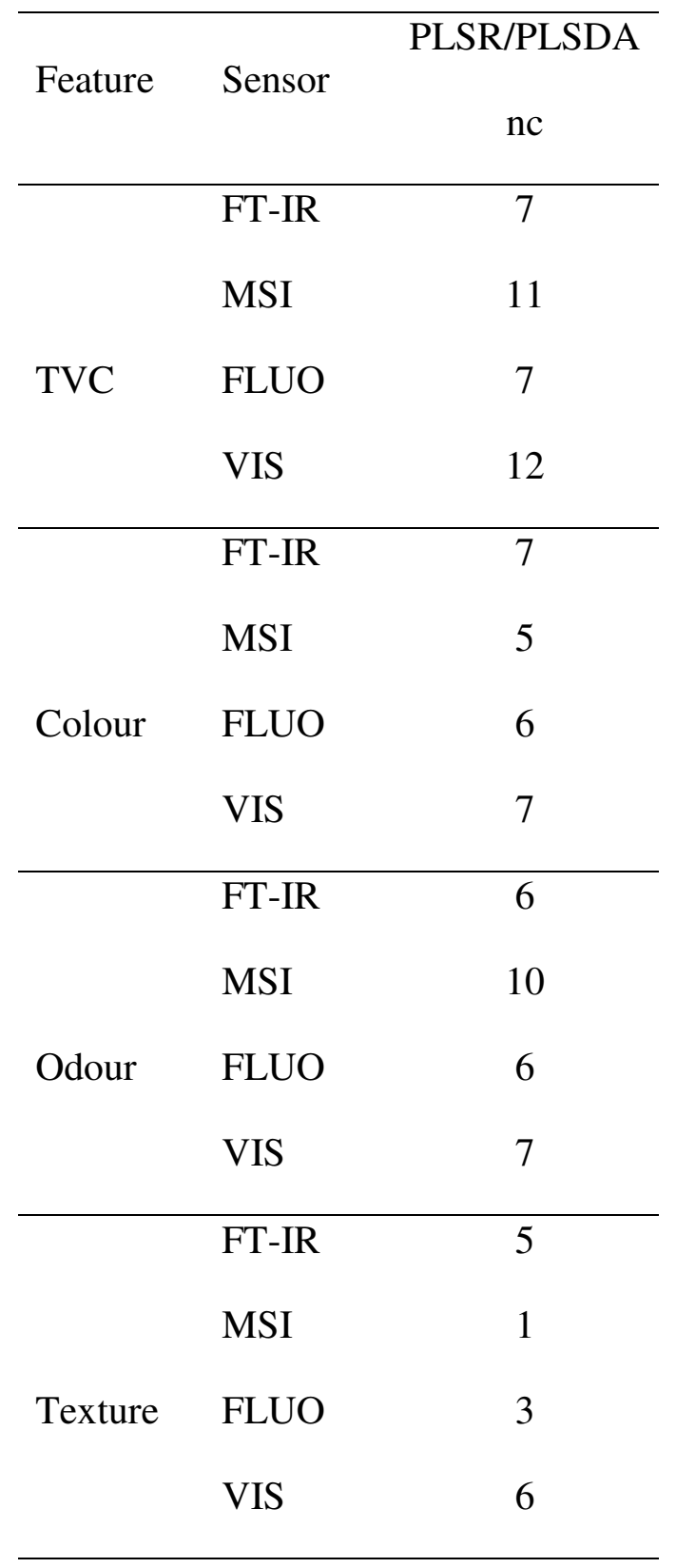

Table 3. Number of latent variables for PLSR and PLSDA models developed for the TVC and sensory features prediction of RTE pineapple on The Unscrambler software. 


\begin{tabular}{|c|c|c|c|c|c|c|c|c|c|}
\hline \multirow{2}{*}{ Model } & \multirow{2}{*}{$\begin{array}{l}\text { Sensory } \\
\text { Feature }\end{array}$} & \multirow{2}{*}{$\begin{array}{c}\mathrm{kNN} \\
\mathrm{k}\end{array}$} & \multirow[b]{2}{*}{ mtry } & \multicolumn{2}{|l|}{ Ranger } & \multirow{2}{*}{$\begin{array}{c}\text { SVM } \\
\text { Linear } \\
\text { c }\end{array}$} & \multicolumn{2}{|c|}{ SVM Radial } & \multirow{2}{*}{$\begin{array}{c}\text { PLSR/ } \\
\text { PLSDA } \\
\text { nt }\end{array}$} \\
\hline & & & & splitrule & $\begin{array}{l}\text { min.no } \\
\text { de.size }\end{array}$ & & sigma & $\mathrm{c}$ & \\
\hline \multirow{4}{*}{ FT-IR } & TVC & 13 & 963 & variance & 5 & 1 & 0.03 & 4 & 7 \\
\hline & Colour & 5 & 7 & extratrees & 1 & 1 & 0.02 & 1 & 8 \\
\hline & Odour & 23 & 620 & gini & 1 & 1 & 0.02 & 128 & 7 \\
\hline & Texture & 9 & 11 & extratrees & 1 & 1 & 0.01 & 0.25 & 7 \\
\hline \multirow{4}{*}{ MSI } & TVC & 17 & 31 & variance & 5 & 1 & 0.05 & 2 & 11 \\
\hline & Colour & 23 & 2 & extratrees & 1 & 1 & 0.05 & 0.25 & 5 \\
\hline & Odour & 11 & 26 & gini & 1 & 1 & 0.06 & 16 & 14 \\
\hline & Texture & 15 & 2 & extratrees & 1 & 1 & 0.06 & 0.5 & 14 \\
\hline \multirow{4}{*}{ FLUO } & TVC & 33 & 122 & extratrees & 5 & 1 & 0.01 & 8 & 7 \\
\hline & Colour & 9 & 67 & gini & 1 & 1 & 0.01 & 0.5 & 7 \\
\hline & Odour & 17 & 57 & gini & 1 & 1 & 0.01 & 4 & 5 \\
\hline & Texture & 11 & 113 & gini & 1 & 1 & 0.01 & 32 & 11 \\
\hline \multirow{4}{*}{ VIS } & TVC & 27 & 94 & variance & 5 & 1 & 0.02 & 4 & 6 \\
\hline & Colour & 5 & 2 & extratrees & 1 & 1 & 0.02 & 0.5 & 5 \\
\hline & Odour & 5 & 104 & gini & 1 & 1 & 0.02 & 16 & 11 \\
\hline & Texture & 5 & 85 & gini & 1 & 1 & 0.02 & 4 & 15 \\
\hline
\end{tabular}

Knn: $k=$ number of neighbours considered,

Ranger: mtry=number of variables to possibly split at in each node, splitrule=splitting rule,

min.node.size= minimal node size,

SVM: $c=$ cost of constraints violation, sigma=scale parameter of the hypothesized (zero-mean) Laplace distribution estimated by maximum likelihood

PLSR/PLSDA: $n t=$ number of components

Table 4. Tuning parameters used in the development of the different models for the TVC and sensory features prediction of RTE pineapple on SorfML software. 


\begin{tabular}{llccc}
\hline Sensor & $\begin{array}{l}\text { Sensory } \\
\text { Feature }\end{array}$ & $\begin{array}{c}\text { Number } \\
\text { of Fresh }\end{array}$ & $\begin{array}{c}\text { Number of } \\
\text { Non-Acceptable }\end{array}$ & $\begin{array}{c}\text { Total } \\
\text { Number }\end{array}$ \\
\hline & Colour & 333 & 91 & \\
FT-IR / & Odour & 147 & 277 & 424 \\
MSI & Texture & 314 & 110 & \\
& Colour & 314 & 78 & 392 \\
$\begin{array}{l}\text { FLUO / } \\
\text { VIS }\end{array}$ & Odour & 143 & 249 & \\
& Texture & 292 & 100 & \\
\hline
\end{tabular}

Table S1. Total number of the measured fresh (acceptable) and non-acceptable samples for each sensory feature. 


\begin{tabular}{|c|c|c|c|c|}
\hline Sensor & $\begin{array}{l}\text { Sensory } \\
\text { Feature }\end{array}$ & Accuracy $(\%)$ & Sensitivity (\%) & Specificity (\%) \\
\hline \multirow{3}{*}{ FT-IR } & Colour & 83.04 & 97.62 & 39.29 \\
\hline & Odour & 76.79 & 50.00 & 84.88 \\
\hline & Texture & 72.32 & 90.00 & 28.13 \\
\hline \multirow{3}{*}{ MSI } & Colour & 85.71 & 100 & 42.86 \\
\hline & Odour & 83.04 & 61.54 & 89.53 \\
\hline & Texture & 72.32 & 95.00 & 15.63 \\
\hline \multirow{3}{*}{ FLUO } & Colour & 84.62 & 93.67 & 56.00 \\
\hline & Odour & 86.54 & 88.46 & 85.90 \\
\hline & Texture & 70.19 & 90.54 & 20.00 \\
\hline \multirow{3}{*}{ VIS } & Colour & 81.73 & 98.73 & 28.00 \\
\hline & Odour & 83.65 & 57.69 & 92.31 \\
\hline & Texture & 68.27 & 90.54 & 13.33 \\
\hline
\end{tabular}

Table S2. Performance metrics of the PLSDA models based on the different analytical platforms (sensors), for the sensory features prediction of RTE pineapple on The Unscrambler software.

\begin{tabular}{llllc||lllll}
\hline Sensor & \multirow{2}{*}{ Algorithm } & $\begin{array}{l}\text { Sensory } \\
\text { Feature }\end{array}$ & $\begin{array}{c}\text { Sensitivity } \\
(\%)\end{array}$ & $\begin{array}{c}\text { Specificity } \\
(\%)\end{array}$ & Sensor & Algorithm & $\begin{array}{l}\text { Sensory } \\
\text { Feature }\end{array}$ & $\begin{array}{c}\text { Sensitivity } \\
(\%)\end{array}$ & $\begin{array}{c}\text { Specificity } \\
(\%)\end{array}$ \\
\hline
\end{tabular}




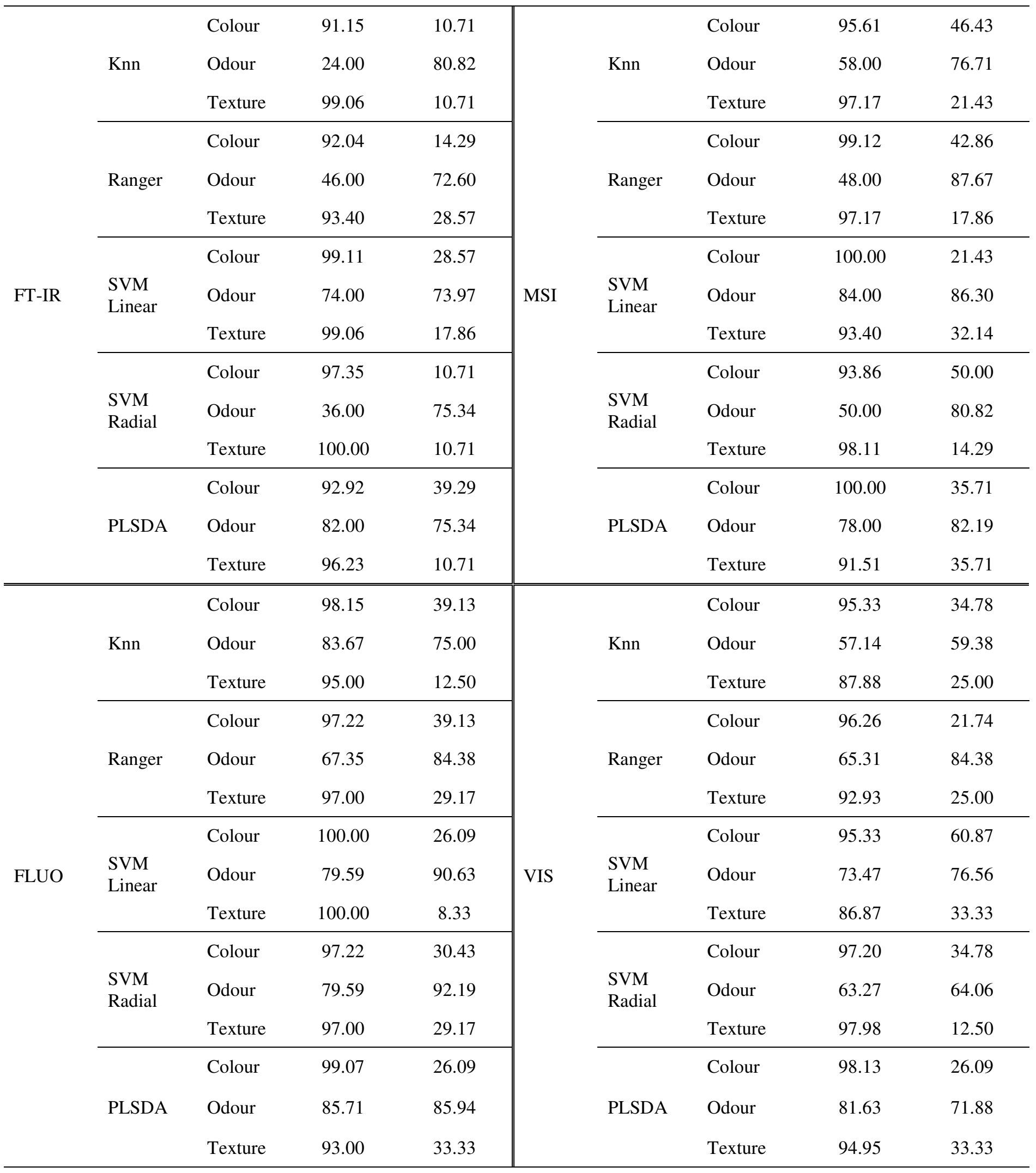

Table S3. The specificity and sensitivity values of the PLSDA models, derived from the various combinations of the different analytical platforms (sensors) and algorithms, for the sensory features prediction of RTE pineapple on SorfML software. The accuracy values are presented in heatmaps. 


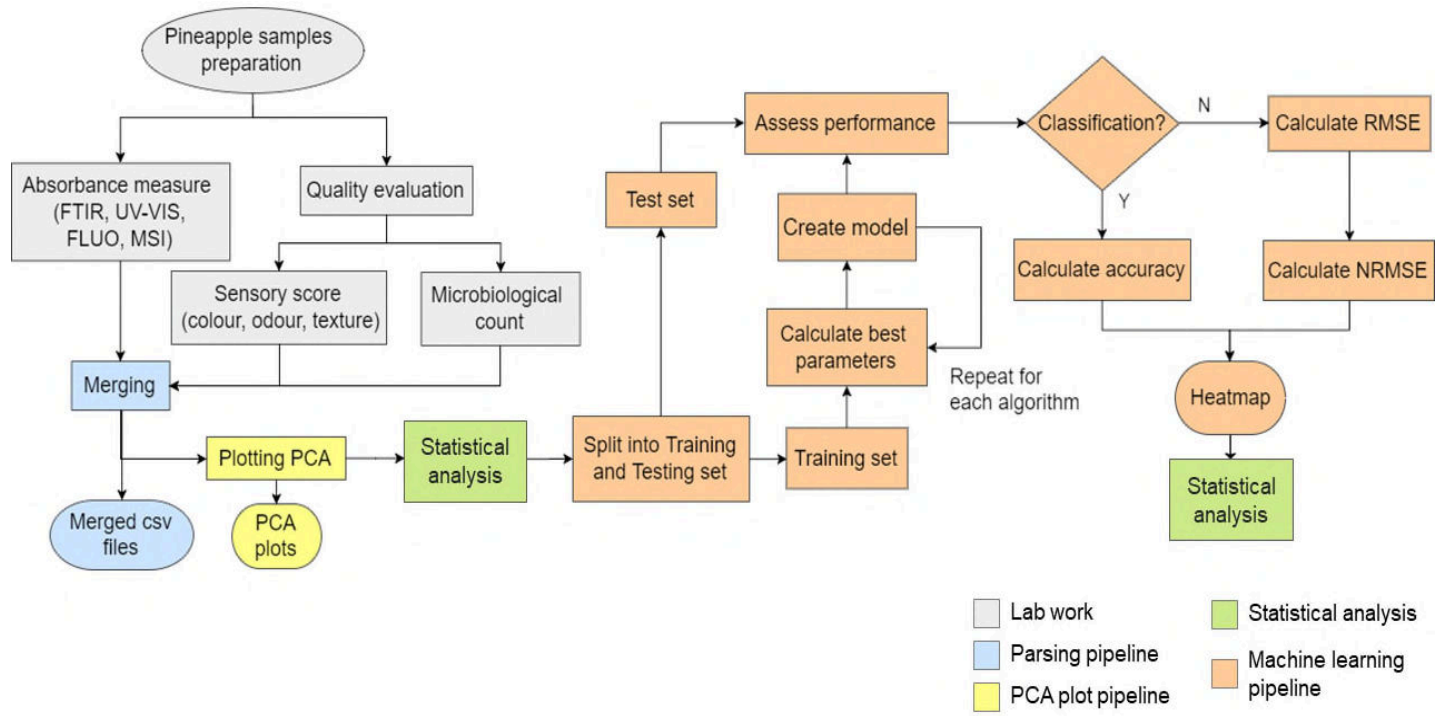

Figure 1. Workflow of SorfML. The workflow is divided into five sections with a different colour according to which part of the methodology the step belongs to. 

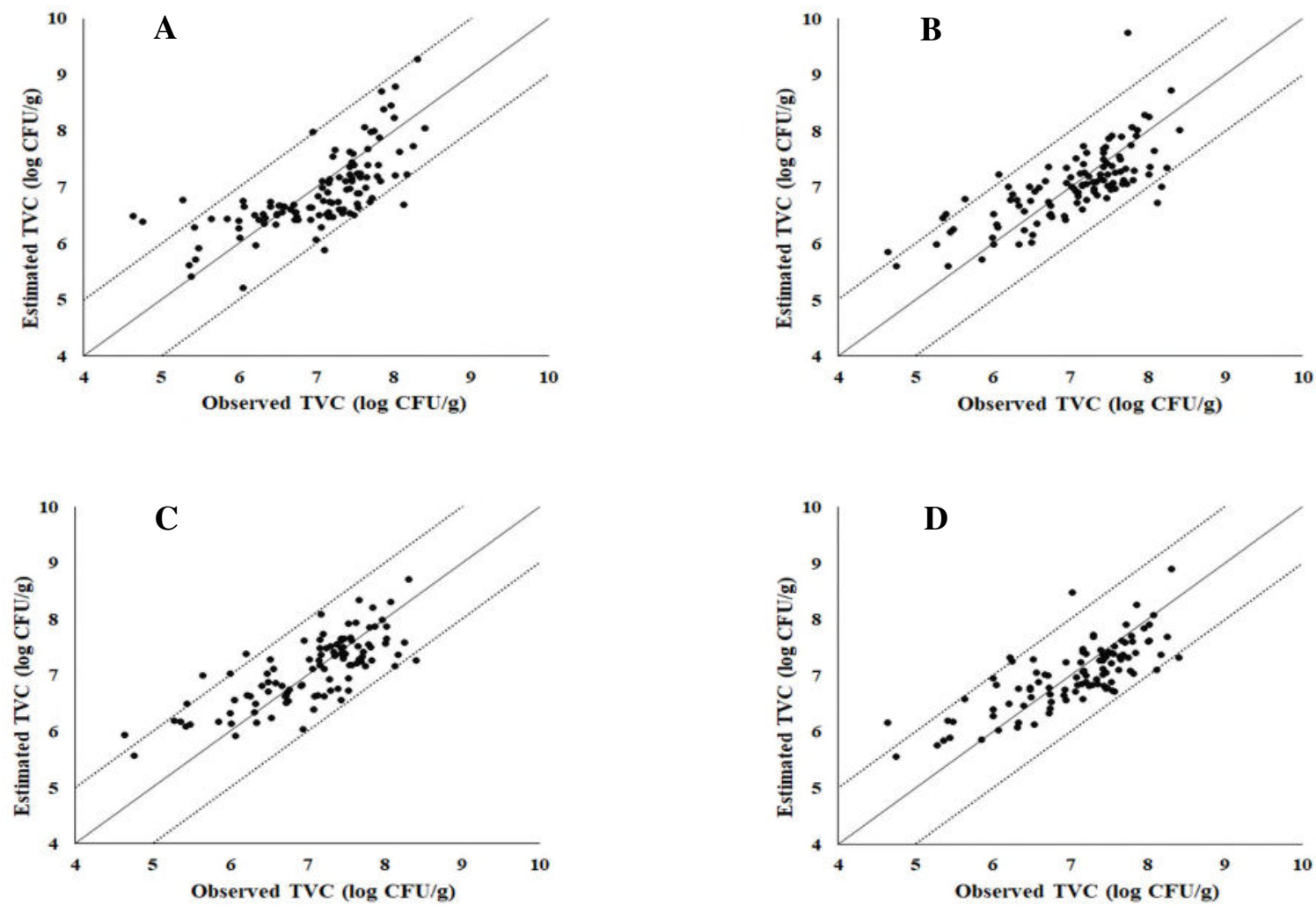

Figure 2. The linear regression between the predicted and the measured by the PLSR model TVC values of prediction based on A) FTIR, B) MSI, C) FLUO, D) VIS data for RTE pineapple, using The Unscrambler software (solid line: the ideal $y=x$ line; dashed lines: the \pm 1 log unit area). 

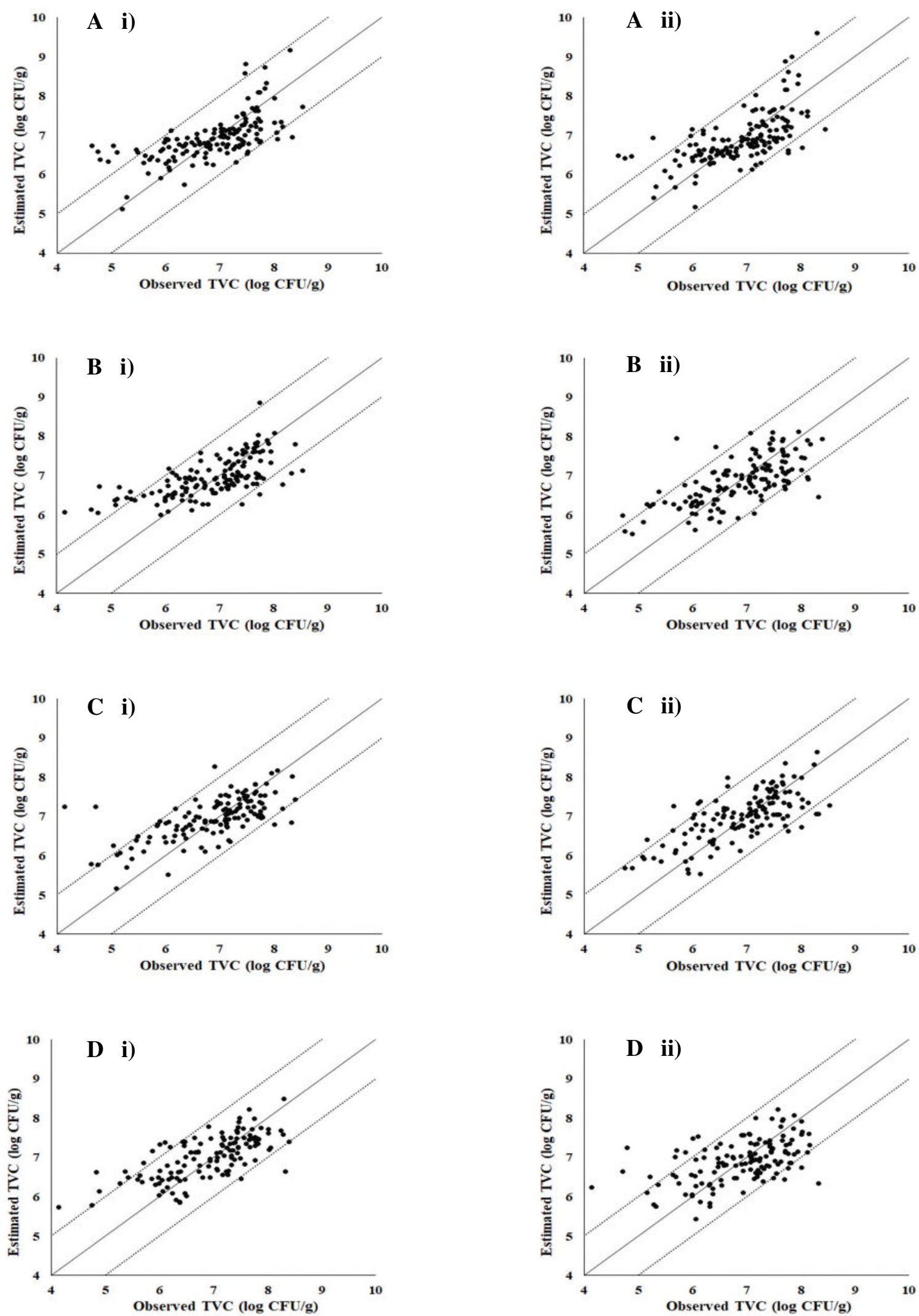
Figure 3. The linear regression between the predicted and the measured by i) SVM Linear and ii) PLSR models TVC values of prediction based on A) FTIR, B) MSI, C) FLUO, D) VIS data for RTE pineapple, using SorfML software (solid line: the ideal $y=x$ line; dashed lines: the $\pm 1 \log$ unit area).
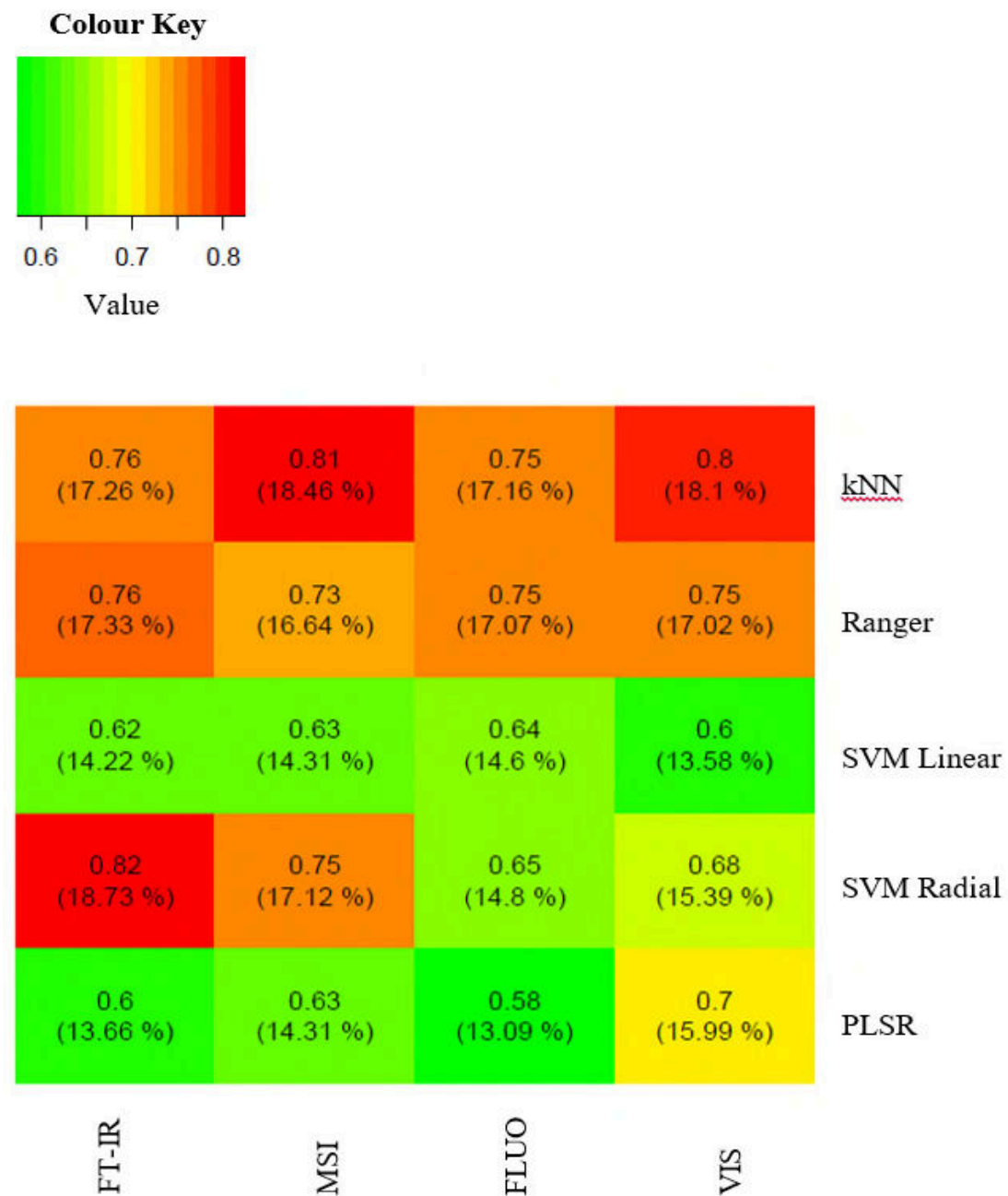

Figure 4. Performance heatmap of the different models developed for each analytical platform for the TVC prediction of RTE pineapple. In each heatmap, the rows belong to the five different algorithms, while the columns are the four different analytical platforms. The RMSE (log CFU/g) and NRMSE (\%) values are presented. The colour key depicts the extreme intensity for the extreme values. The colour key begins from green (higher performance) to red (lower performance). 

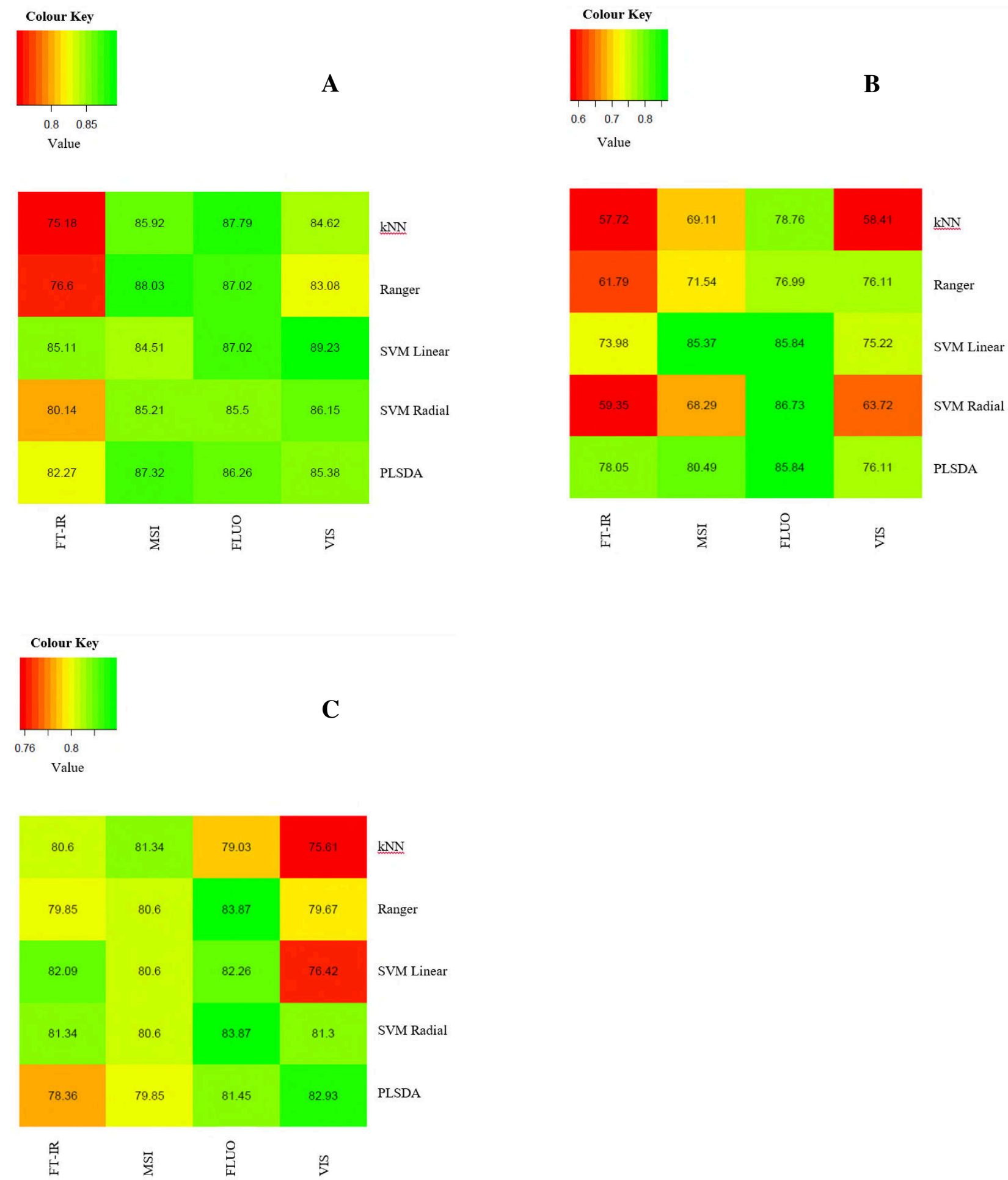

Figure S1. Performance heatmaps of the different models developed for each analytical platform for the A) Colour, B) Odour and C) Texture prediction of RTE pineapple. In each heatmap, the rows belong to the five different algorithms, while the columns are the four different analytical platforms. The accuracy (\%) values are presented for the sensory features. The colour key depicts the extreme intensity for the extreme values. The colour key begins from red (lower performance) to green (higher performance). 